Service social

\title{
Réflexion sur la condition de la mère de l'enfant handicapé : une intervention féministe à développer
}

\section{Sylvie Tétreault et Linda Blanchette}

Volume 40, numéro 2, 1991

Formation et évolution de la pratique en travail social

URI : https://id.erudit.org/iderudit/706531ar

DOI : https://doi.org/10.7202/706531ar

Aller au sommaire du numéro

Éditeur(s)

École de service social de l'Université Laval

ISSN

1708-1734 (numérique)

Découvrir la revue

Citer cet article

Tétreault, S. \& Blanchette, L. (1991). Réflexion sur la condition de la mère de l'enfant handicapé : une intervention féministe à développer. Service social, 40(2), 117-143. https://doi.org/10.7202/706531ar
Résumé de l'article

Aujourd'hui, la plupart des enfants handicapés demeurent dans leur famille et suivent activement un programme de réadaptation. Souvent, la mère devient la principale intervenante auprès de l'enfant. Mais rapidement, elle se sent essoufflée, exténuée et fatiguée. Cet article propose une réflexion féministe de la maternité et du vécu de la femme ayant un enfant handicapé. Il sera intéressant de voir comment s'est faite la transition de l'institution à la prise en charge des soins de l'enfant handicapé par la mère et d'évaluer le rôle des politiques sociales à ce chapitre. Par la suite, une intervention féministe à préconiser auprès de ces femmes sera proposée aux travailleuses sociales. 


\section{COMMENTAIRES \\ ET DOCUMENTS}

Sylvie Tétreault, étudiante au doctorat en service social et chargée de cours au programme d'ergothérapie, Université Laval.

Linda Blanchette, agente en relations humaines au Département de néonatalogie de l'Hôpital de Montréal pour enfants.

\section{Réflexion sur la condition de la mère de I'enfant handicapé : une intervention féministe à développer}

Peu d'informations existent au Québec sur la mère de l'enfant handicapé. La plupart des connaissances proviennent des recherches américaines ou anglo-saxonnes. Ces études portent principalement sur l'adaptation de la mère à son enfant et les interactions qui existent entre eux. Cependant, certaines données statistiques sont disponibles. Ainsi, les chiffres compilés au Québec sont éloquents et soulignent l'urgence d'agir auprès de ces femmes. Celles-ci remplacent graduellement les institutions asilaires qui accueillaient autrefois les enfants ayant une déficience. Par exemple, Lamarche (1987) rapporte que $5,5 \%$ des enfants qui naissent dans cette province sont handicapés, que ce soit sur le plan physique, intellectuel ou sensoriel. Plus de 277445 enfants canadiens âgés entre 0 et 14 ans présentent un certain degré d'incapacité. Ce qui est encore plus intéressant, c'est que 
$99 \%$ d'entre eux vivent dans leur famille (Statistique Canada, 1988). II apparaît clairement que la tendance à institutionnaliser l'enfant handicapé est carrément révolue. En effet, malgré I'avancement technologique, les changements de mentalité dans la société et le nombre croissant de ressources disponibles pour les personnes handicapées, la famille naturelle se retrouve souvent seule à assumer l'entière responsabilité des soins de l'enfant handicapé, peu importe les coûts financiers et sociaux qu'elle doit payer (Browne, Kirlin et Watt, 1981). Et qui pense famille songe surtout à la femme qui prend en charge les soins de son enfant handicapé.

Est-ce que la société a tendance à encourager la femme ayant un enfant handicapé à correspondre au modèle traditionnel de mère ? La pratique actuelle du service social favorise-t-elle le maintien de la femme dans un rôle passif de mère ? Qui s'inquiète de l'isolement sociétal que vivent ces femmes ? Voilà autant de questions que le présent article peut susciter chez les lecteurs. Précisons que ce texte n'a pas la prétention de répondre à toutes ces interrogations, mais plutôt qu'il vise à mettre en lumière la situation particulière de la femme ayant à sa charge un enfant handicapé. D'abord, pour atteindre cet objectif, un survol de l'analyse féministe de la maternité permettra de mieux saisir le contexte actuel. Par la suite, l'arrivée de l'enfant handicapé et son effet sur la mère seront abordés. Une synthèse de la transition du modèle institutionnel à celui du maintien à domicile sera présentée, de même que l'influence de la législation et des politiques sociales sur ces orientations. Le rôle de l'Office des personnes handicapées du Québec sera analysé. Également, un aperçu rapide de la pratique sociale auprès de cette clientèle sera proposé avant la présentation d'une réflexion portant sur l'approche féministe.

\section{Analyse féministe de la maternité}

\section{Modèles idéologiques de la maternité}

Selon des auteures féministes (Levine et Estable, 1981), il existe quatre éléments qui sont interreliés et qui définissent la maternité en tant qu'institution sociale. II s'agit du patriarcat, des systèmes économiques, de l'institution de la maternité et de l'idéologie de l'amour romantique. Ce sont ces éléments qui ont modelé la perception qu'ont la femme et son environnement des rôles attribués à la mère. Pour mieux comprendre ce que représente la maternité, une description sommaire de ces éléments est proposée.

Ainsi pour Levine et Estable (1981), le patriarcat se définit comme un ensemble de structures, érigé par les hommes, qui contribue à la 
domination des femmes à travers la famille et le travail. L'homme détermine le rôle de la femme en lui demandant d'être passive, dépendante, gentille et dévouée à son mari et à ses enfants. Levine et Estable (1981) blâment les différents systèmes économiques présents d'exploiter les femmes sous tous les aspects de leur vie. En examinant le système capitaliste, les femmes représentent une main-d'œuvre de réserve, mal rétribuée, qui est utilisée selon les besoins de la société. Un exemple de ce phénomène est l'arrivée des femmes sur le marché du travail par suite d'un besoin de main-d'œuvre durant la guerre et leur congédiement au retour des soldats. Quant à l'institution de la maternité, elle maintient les femmes au service des hommes et des enfants. Par le fait même, la maternité contribue à diminuer la place de la femme dans le domaine public. Pour sa part, l'idéologie de l'amour romantique aveugle la femme et sert à perpétuer une répartition inégale du pouvoir entre les sexes. Toujours selon les mêmes chercheuses, la vision romanesque de la vie familiale ne reflète pas la réalité, car elle véhicule une impression permanente de sécurité émotionnelle et financière ainsi qu'une prise en charge de sa vie par l'être aimé. Cette idéologie ne prend pas en considération les besoins individuels de la femme. Les institutions de la maternité et du mariage confinent les femmes aux rôles traditionnels et peuvent les isoler du pouvoir.

Un autre élément qui empêche la femme d'atteindre le pouvoir est la notion de la carence maternelle, développée par Bowlby (1969). Cet auteur suppose que l'absence de la mère auprès de son enfant, particulièrement durant la première enfance, pourrait être la cause de certains problèmes socio-affectifs chez les enfants. D'autres auteurs (Klaus et Kennell, 1972) ont appuyé ces affirmations en spécifiant que la période précédant la naissance de l'enfant constitue une étape importante pour l'attachement maternel. Ils croient qu'une séparation prématurée $d^{\prime}$ avec la mère peut avoir des conséquences importantes sur le développement futur du nourrisson. Par exemple, l'apparition de dépression, de délinquance et d'autres atteintes à la santé mentale peut s'expliquer par l'expérimentation chez ces enfants d'une carence maternelle. Les féministes précisent que cette notion est une forme déguisée d'antiféminisme. Selon le discours véhiculé par Levine et Estable (1981), cette théorie amène la femme à se sentir responsable des comportements de son enfant. Ces auteures sont conscientes que les enfants ont besoin de soins, d'amour et de tendresse dans une relation continue avec une personne, peu importe son sexe. Mais, elles s'opposent à ce que les soins de l'enfant soient la responsabilité entière de la femme. Elles $s^{\prime}$ interrogent sur le fait que le rôle du père n'a pas été considéré par Bowlby au cours de ses études sur la carence maternelle. De plus, elles reconnaissent les institutions et les structures sociales comme façonnant le comportement humain et empê- 
chant les femmes de développer leur propre identité. Ainsi la cellule familiale traditionnelle serait maintenue en place au détriment de l'autonomie de la femme.

En résumé, l'approche féministe critique le fait que la maternité peut devenir un carcan pour la femme en ce sens qu'elle l'amène à consacrer une grande partie de son énergie à son rôle de mère, tout en négligeant ses propres besoins. Les femmes évaluent continuellement leurs qualités de mère en fonction d'objectifs impossibles à atteindre et elles s'excusent sans cesse pour les fautes de leurs enfants. L'institution de la maternité a établi un modèle de la mère qui frôle l'excellence (Levine et Estable, 1981). En tenant compte de cette perspective féministe, il s'avère essentiel d'analyser comment se déroule l'attente d'un enfant et de voir son influence sur le développement de l'identité comme mère.

\section{Attente d'un enfant}

Actuellement, la société québécoise s'inquiète devant la situation de la dénatalité, ce qui contribue à valoriser la maternité. Par le fait même, le rôle de mère est idéalisé et la femme doit jouer ce rôle à la perfection. Une mère se considère et est considérée par les autres comme étant responsable de la qualité de vie de son enfant. De plus, une fois la grossesse terminée, la femme porte socialement le poids de l'engagement à long terme encore plus que l'homme. Celui-ci peut participer aux soins de l'enfant s'il le désire. La femme, elle, s'engage davantage et la responsabilité de la garde des enfants lui incombe presque toujours après une séparation (Ross, 1983). L'existence même de l'enfant peut envahir celle de la mère, car elle se doit de produire un enfant qui est acceptable pour la société (Thorne et Yalom, 1982). Malgré ces pressions, l'attente d'un enfant est teintée de fierté, d'espoir et d'aspirations diverses. La maternité devient un rite de passage dans le clan des mères qui favorise l'apprentissage d'un nouveau rôle social (Quéniart, 1989a).

Avant sa naissance, le bébé est imaginé par la mère. Elle le visualise avec toute la fantaisie de l'enfant parfait. Malheureusement pour elle, il n'y a pas de garantie que le bébé soit normal, qu'il corresponde à ses désirs. La femme devient alors hypersensible et vulnérable durant sa grossesse, surtout à cause de l'insécurité qu'elle vit par rapport à la possibilité d'avoir un bébé handicapé (Ross, 1983). La peur des femmes est d'autant plus grande qu'elles ne savent plus trop où commencent et où s'arrêtent les risques encourus. Cette peur peut devenir tellement grande qu'elles considèrent la normalité du fœtus comme le seul élément important de leur grossesse (Quéniart, 1989a). Pour diminuer les craintes de la future mère, le diagnostic prénatal est très popu- 
laire. Cette technologie renforce l'entente tacite qui exige des femmes qu'elles produisent un enfant en bonne santé et qui devient un moyen pour garantir le bébé parfait. Cependant, il ne faut pas se leurrer, car la technologie ne permet pas d'éliminer les handicaps, mais seulement de les dépister (Quéniart, 1989b). Toute cette technologie (qui comporte la notion de fabrication) devient pour la femme le seul moyen d'être rassurée, de savoir si tout va bien et surtout de repérer l'éventuelle anomalie. Ce sont des moyens (qui sont encore sous le contrôle de la médecine) pour garantir le bébé parfait que désirent toutes les femmes. Nous sommes passés du désir de l'enfant au droit de choisir quand l'avoir, puis au droit d'avoir un enfant " normal ». Tout cela conduit la femme à vouloir reproduire un être conforme aux demandes de la société, normalisé et standard (Quéniart, 1989a, b).

À I'heure du diagnostic prénatal, on parle peu du risque d'erreur, de la possibilité d'eugénisme de la part de la société, et l'on oublie que $\mathrm{s}^{\prime}$ il y a un problème, c'est la femme qui doit prendre la décision de poursuivre ou non la grossesse (Quéniart, 1989b). Selon Dallaire (1982; voir Saillant et $\mathrm{O}^{\prime}$ Neill, 1987) si un diagnostic par amniocentèse met en évidence une anomalie, dans $99 \%$ des cas la mère choisit l'avortement et cela quelle que soit l'anomalie détectée. II faut s'inquiéter à savoir jusqu'où cette tendance se poursuivra avec des handicaps plus légers. Jean (1987) mentionne que des avocats américains considèrent que la femme qui n'aurait pas d'interruption de grossesse à la suite de l'annonce d'un fœtus anormal pourrait avoir des poursuites judiciaires. Sa décision serait perçue comme de la négligence envers l'enfant à naître. À un autre niveau du débat, une société qui valoriserait à outrance la naissance d'enfants normaux et dont les standards de normalité seraient définis très clairement pourrait exiger d'une femme enceinte de suivre un régime de vie approprié. Avec un peu d'imagination, il serait possible de voir apparaître des interdictions de vente de cigarettes ou d'alcool à la femme enceinte.

\section{Arrivée de l'enfant handicapé}

\section{Le choc de la naissance}

Devant toutes ces pressions vers la normalité, comment une femme réagit-elle à l'arrivée de l'enfant handicapé ? L'analyse de Butani (1970) indique que lorsqu'un bébé naît avec un handicap ou une tare génétique, la mère est vivement déçue, dévalorisée, lésée. Tous les rêves et les aspirations faits durant la grossesse disparaissent de façon tragique, car aucune personne n'est jamais assez préparée pour devenir subitement parent d'un enfant handicapé. Cet événement 
douloureux perturbe grandement toute la famille et la plonge dans une situation de crise intense qui demande des ajustements psychologiques (Lonsdale, 1978; Mori, 1983). De la peur et de l'anxiété peuvent apparaître, surtout si la vie de l'enfant est en danger et que la mort est envisagée par l'équipe médicale (Mattsson, 1980). La réponse initiale se caractérise par un état de choc, qui est suivi de déni et de tristesse. Après une période de réajustement, la mère doit faire face aux problèmes quotidiens (Gath, 1977). Plus la malformation est apparente, plus la réaction de la mère sera rapide. Elle se perçoit inadéquate devant ce bébé qui ne correspond pas à ses aspirations. Son idéalisation de l'enfant est détruite en peu de temps. Elle se sent vide et seule. Quant à Burley (1981), il reconnaît que la connaissance du diagnostic devient un événement tragique qui affecte toute la famille, mais spécialement la mère. Pour lui, la femme se considère comme encore plus responsable de la situation que les autres membres, car elle considère le bébé comme le prolongement d'elle-même. La mère se demande quelle faute elle a pu commettre durant la grossesse pour avoir un tel enfant. Elle cherchera toujours les raisons qui ont pu provoquer le handicap de son enfant.

Les mères se sentent différentes et elles vont s'isoler facilement (Shapiro, 1981). La plupart des réactions émotionnelles de la mère sont liées à l'acceptation et au soutien de la société à l'égard de son enfant handicapé. La mère se sentira inférieure pour avoir produit un tel enfant et elle pourra éprouver une peur d'être rejetée par les autres (Gordeuk, 1976). Toute l'énergie engendrée par le désappointement et la frustration d'avoir mis au monde un enfant handicapé se transformera en colère et en hostilité. Pour la mère, le handicap peut être perçu comme une punition personnelle. Afin de parvenir à l'acceptation de son enfant tel qu'il est, la mère doit cheminer à travers le processus de deuil de l'enfant parfait. Elle passe par une période de choc, de négation, de tristesse, de rage et d'anxiété. Elle développe des sentiments de culpabilité et s'interroge sur sa capacité d'assumer ces sentiments et la situation générale (Butani, 1970).

\section{Atteinte de la santé physique et mentale de la mère}

Un sentiment de perte de l'enfant idéal, combiné à un manque de préparation face à cet événement, perturbe grandement la santé mentale autant que la santé physique de la femme (Burley, 1981). Plusieurs études (Breslau, Staruch et Mortimer, 1982; Burden, 1980; Chetwynd, 1985; Kemp et Hatmaker, 1989; Shapiro, 1989) ont démontré de façon significative que la femme ayant un enfant handicapé éprouve plus de stress que la mère d'un enfant non handicapé. À long terme, un tiers des mères d'enfants handicapés connaissent des problèmes psychi- 
ques et somatiques. Dépression, fatigue, épuisement, douleurs musculaires au dos et troubles du sommeil constituent quelques-uns des symptômes vécus par la mère (Zucman, 1982). Les problèmes de dos sont causés par les nombreuses manipulations de l'enfant et apparaissent comme la plainte principale des mères (Burden, 1980). Ces données correspondent à l'étude de Lonsdale (1978), qui affirme que $33 \%$ des mères d'enfants handicapés présentent des malaises d'origine psychologique ou physique. II reconnaît que lorsque la mère doit encore se lever la nuit, cela contribue à altérer sa santé, à accroître sa fatigue et son insomnie. Les mères ont souvent tendance à imputer leurs sentiments dépressifs à un désordre hormonal, à des problèmes conjugaux ou à leur isolement social dû à leur condition de ménagère plutôt que de les attribuer à la présence de l'enfant handicapé. Une grande proportion d'entre elles rapportent être très nerveuses et ce sont surtout les mères monoparentales qui avouent utiliser des tranquillisants (Doner, 1975).

\section{Répercussions sur l'emploi}

Un autre aspect de la vie de la femme qui est touché par la présence d'un enfant handicapé est l'accessibilité pour elle au marché du travail. Il s'agit d'un point important à aborder, car les possibilités financières de la mère sont intimement liées à sa situation de vie. Ce n'est pas un choix facile pour la femme d'aujourd'hui qui, d'une part, fait face à de nouvelles perspectives d'emplois, de carrière, d'ouverture dans des sphères autrefois réservées uniquement aux hommes et, d'autre part, ressent en même temps les pressions de l'environnement et de la société pour qu'elle demeure à la maison avec son enfant handicapé. C'est dire que l'arrivée d'un enfant handicapé a des répercussions importantes sur l'activité professionnelle de la mère. Plusieurs facteurs identifiés par Breslau (1983) favorisent I'abandon du travail à l'extérieur par la mère. Il s'agit des caractéristiques de la famille comprenant sa composition et l'âge des autres enfants, le type d'emploi occupé par la femme, le niveau socio-économique de la famille incluant son revenu, sa culture et son degré de scolarité. Ainsi, dans de nombreux cas, la mère quitte son emploi pour se consacrer presque exclusivement à son enfant handicapé, souvent au détriment des autres enfants (Zucman, 1982). Cela a aussi des répercussions sur ses possibilités d'avoir une scolarité plus avancée, un revenu plus élevé et une carrière professionnelle (Breslau, 1983). D'ailleurs, Norman et Mancuso (1980) constatent eux aussi que la femme retourne à la maison plus facilement que son mari pour s'occuper de son enfant handicapé. Cela est attribuable à son salaire plus faible et au type d'emploi occupé qui est souvent peu valorisé par le conjoint. Selon Breslau, Staruch et 
Mortimer (1982), le fait que la femme reste confinée à un rôle de gardienne d'enfant et qu'elle ne peut aller travailler constitue un facteur de risque important de dépression ainsi que de détresse psychologique. Plus l'enfant sera dépendant, plus grande sera la détresse psychologique. Et même si la mère trouve un emploi à l'extérieur de la maison, elle demeure la principale responsable de l'entretien ménager et des soins à donner à l'enfant handicapé (Shapiro, 1989).

\section{Répercussions sur la vie conjugale}

Tous ces événements placent le couple dans une situation angoissante. II ne faudrait pas négliger, comme le souligne McCubbin (1989), l'importance du soutien de la part du conjoint afin de diminuer l'effet du stress sur la mère et de permettre la mise en place de mécanismes d'adaptation pour faire face à l'acceptation de l'enfant handicapé. Même si les deux parents sont affectés par cette naissance, le stress est toujours plus grand sur la mère que sur le père et encore plus important s'il s'agit d'une mère sans conjoint. La présence continuelle de l'enfant handicapé accentuera la disparité des rôles entre eux (Smith, 1986). Selon Handleman et Harris (1986), cette différence est due au fait que le père joue un rôle moins central que la mère par rapport aux soins des enfants et que cette dernière s'implique très tôt comme parent. À cet effet, Mardinos (1985) souligne que les mères ne veulent pas imposer de fardeau supplémentaire à leur conjoint et, de ce fait, préfèrent ne pas leur confier leurs préoccupations quotidiennes. Elles tentent plutôt de régler elles-mêmes les problèmes. L'auteure fait également ressortir que plus les femmes assument de responsabilités, plus les conjoints adoptent des attitudes masculines traditionnelles et qu'ils prennent des distances par rapport à la famille.

À court terme, le père se détache émotionnellement de l'enfant, forçant encore plus la mère à s'en occuper totalement. II concentre toute son énergie dans son travail à l'extérieur de la maison ou dans des activités sociales (Kazak et Marvin, 1984; Mori, 1983; Shilling, Kirkham, Snow et Schinke, 1986). Ces constatations sont appuyées par différents écrits (Holroyd, 1974; Kazak et Marvin, 1984; Smith, 1986). Comparativement au père, la mère dispose de très peu de moments de liberté ou de loisir. McCubbin (1989) explique cela par la présence à l'intérieur de ces couples de la répartition traditionnelle des tâches, qui suppose que c'est principalement la mère qui s'occupe des enfants. Pourtant, cet auteur relève dans son étude que le partage des responsabilités est un élément important de la satisfaction maritale, qui, à son tour, est le meilleur prédicteur de l'habileté psychologique de la mère à faire face au stress et à s'ajuster à la prise en charge de l'enfant handicapé. 
Un cercle vicieux se crée où la mère est débordée par ses tâches quotidiennes, ce qui l'amène à investir peu auprès de son conjoint, qui à son tour se détache graduellement du foyer familial. II s'ensuit une mise à l'épreuve quotidienne des liens du couple. Zucman (1982) suggère que le handicap de l'enfant peut devenir un révélateur des faiblesses ou des potentiels du couple. La présence de l'enfant handicapé cristallise les conflits dans le couple. II n'est donc pas étonnant, selon Davies (1982), de noter une augmentation du divorce chez ces personnes. Plusieurs divorces peuvent être attribués à la différence entre les conjoints en ce qui a trait à leur acceptation du handicap de l'enfant. Celui-ci peut devenir une excuse pour faciliter la séparation à l'intérieur d'un couple qui avait déjà des difficultés avant son arrivée. Le fait que le stress vécu par les conjoints soit distribué inéquitablement entre eux constitue un autre élément favorisant la rupture (Vash, 1981).

En somme, Burden (1980) affirme que la mère de l'enfant handicapé a des besoins spéciaux et que la société doit répondre à ses demandes afin de lui éviter l'épuisement chronique et les problèmes de santé mentale. C'est à partir de ces constatations que la figure qui apparaît à la page suivante a été bâtie. Elle décrit le vécu de la mère à travers les sentiments qu'elle éprouve et les événements auxquels elle doit faire face.

Cette figure tente de démontrer que la femme fait face à une variété de sentiments, de situations et de choix qui bouleversent son équilibre autant psychique que physique. Ce déséquilibre est accentué par le manque de ressources d'aide et par les luttes incessantes qu'elle doit mener pour recevoir des services. Le surcroît de travail est aussi causé par les nombreuses demandes en provenance de la famille et des milieux spécialisés. II n'est donc pas étonnant qu'une désorganisation de l'unité familiale puisse se produire. Tout cela est associé à la dépendance de la femme à l'égard des politiques sociales qui accélèrent son isolement.

Après ce constat du vécu de la mère de l'enfant handicapé, plusieurs observations semblent ressortir. Des barrières sociales, psychologiques et culturelles entourent la femme-mère, limitent ses aspirations, contribuent à la rendre responsable du handicap de l'enfant et de son niveau de développement. Ces barrières amènent la femme à croire qu'elle doit rester à la maison pour s'occuper de l'enfant, car personne d'autre ne peut la remplacer. Sa présence devient essentielle. Mais comment la femme en arrive-t-elle à ces pensées ? Comment peut-elle arriver à vivre dans de telles conditions ? Quels sont les éléments qui ont contribué à augmenter le poids de la prise en charge des soins de l'enfant handicapé ? La prochaine section de cet article tente de mieux comprendre l'évolution de la prise en charge des soins de l'enfant handicapé. 
FIGURE 1

Le vécu de la mère de l'enfant handicapé

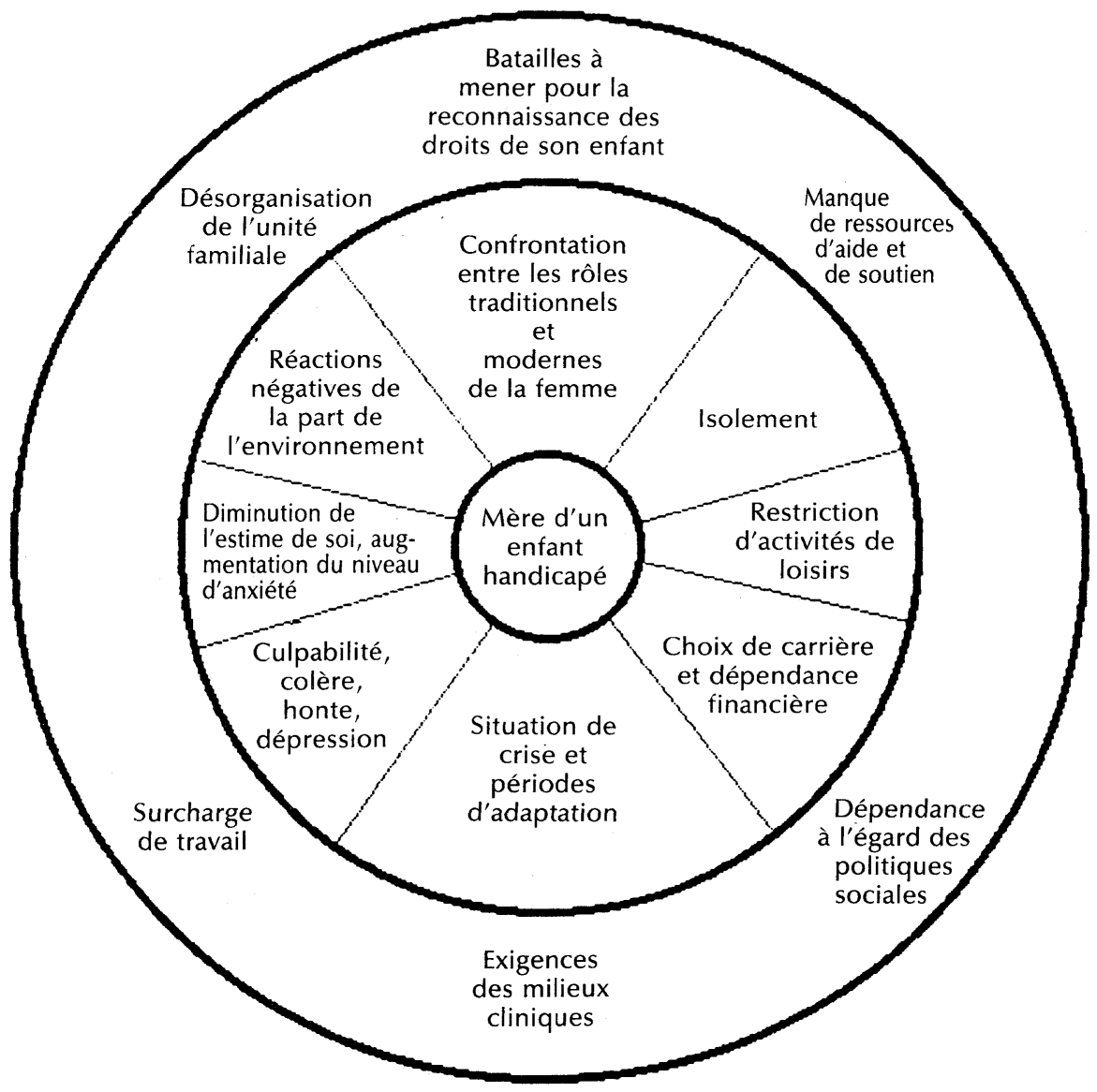

\section{Évolution de la prise en charge}

\section{De l'institution à la maison}

L'enfant handicapé n'a pas toujours été confié immédiatement à ses parents. En effet, au tout début de la colonie, les personnes inaptes au travail (orphelins, infirmes et personnes âgées) étaient souvent abandonnées et recueillies dans les hôpitaux généraux. Après la conquête, les nouveaux dirigeants cessèrent de consacrer de l'argent à I'entretien des enfants abandonnés. Cette œuvre fut alors prise en 
charge par la communauté des Sœurs Grises (fondée en 1738) jusqu'au milieu du XXe siècle (Lapointe-Roy, 1987). Les congrégations religieuses ont été longtemps les principales ressources pour les familles des personnes handicapées. Avec les organismes bénévoles, elles ont été les seules à fournir des soins aux personnes malades avant que l'intervention de l'État ne devienne de plus en plus présente (OPHQ, 1987). La religion, surtout au Québec, a été un élément qui encourageait les femmes à accepter leur enfant handicapé comme un don de Dieu, même si la plupart des gens associaient le handicap à une punition ou à un signe du démon. Cette croyance est encore très ancrée au Québec (Lamarche, 1987).

À cette époque et pendant de nombreuses années, la population d'enfants handicapés fut très limitée car il existait une élimination naturelle de ces enfants. Comme ils étaient de constitution faible, les maladies infantiles ou de nombreuses épidémies les disséminaient facilement (Lapointe-Roy, 1987). Cook (1988) mentionne que les survivants étaient confinés dans des sanatoriums, qu'ils étaient bannis de la vie communautaire à tout jamais. Ce mouvement $d^{\prime}$ 'institutionnalisation et d'internement était généralisé à toutes les personnes différentes, peu importe leur âge ou leur handicap. Celles-ci étaient enfermées avec d'autres individus qui représentaient une menace pour la société d'alors, soit par leurs comportements (ex. : folie, violence, crime), leur état (ex. : maladie contagieuse, pauvreté, débauche) ou à cause de leurs idées (ex. : opinions politiques marginales) (Boudreau, 1984). Peu sensibilisés aux possibilités et aux capacités des personnes handicapées, les médecins les dirigeaient automatiquement vers des centres d'accueil ou des établissements de soins psychiatriques. Ces individus étaient pratiquement relégués aux oubliettes (OPHQ, 1982).

Au tout début du XX $X^{e}$ siècle, I'urbanisation a favorisé le départ des familles vers la ville. La femme a commencé à travailler à l'extérieur de la maison et la plupart des personnes handicapées n'avaient maintenant plus de place dans la famille. L'institutionnalisation se faisait régulièrement en cachette de la nouvelle mère. Très souvent, après l'accouchement, les autorités médicales convainquaient le père de ne pas montrer l'enfant handicapé à la mère et de l'envoyer immédiatement dans une institution. Ils recommandaient à la famille et surtout à la mère d'oublier à jamais cet enfant. Cette mise à l'écart de la personne handicapée de la société existera encore dans les années soixante. En effet, De Billy (1984) soutient que la naissance d'un enfant handicapé demeurait entourée de secret. Souvent, l'enfant était immédiatement placé par la famille, qui tentait de l'oublier et de ne plus en parler. Une minorité de parents gardaient l'enfant à la maison. Cependant, certains des enfants qui demeuraient avec leur famille vivaient dans des conditions d'exclusion de la vie communautaire ou familiale. 
Ils étaient isolés et cachés loin de toute stimulation (OPHQ, 1987). De Billy (1984) mentionne qu'aujourd'hui la situation est inversée, mais que ces changements dans les mentalités ne se sont pas faits sans heurt. D'ailleurs, quelques parents (Burley, 1981; Desroches-Oligny, 1980; Simons, 1987) ont décrit leur cheminement dans la littérature. Ces parents indiquent qu'ils sont livrés à eux-mêmes et qu'ils doivent tenter de découvrir les ressources au moment même où ils ont le plus besoin de soutien.

Il faut mentionner l'importance de la période qui suit la Première Guerre mondiale dans la reconnaissance des droits des enfants handicapés. Au cours de ces années, une nouvelle vision de la réadaptation se développera pour faire face aux déficits causés par les blessures de guerre (OPHQ, 1982). Graduellement, les enfants pourront bénéficier des nouvelles technologies utilisées dans ces centres de réadaptation (par exemple le développement de nouvelles prothèses pour les amputés de guerre). De plus en plus, la situation favorise l'émergence des besoins des autres groupes de personnes handicapées et accentue les pressions pour faire reconnaître leurs droits à des services spécialisés et à une participation sociale accrue (OPHQ, 1987). Tout ce mouvement convainct graduellement la famille et surtout la mère du bienfondé de garder l'enfant handicapé à la maison après sa naissance. Maintenant, il est logique et socialement acceptable que la mère, à la suite de la naissance d'un enfant handicapé, quitte son emploi pour rester à la maison et s'en occuper (Mardinos, 1985). Plusieurs facteurs peuvent expliquer le maintien à domicile de l'enfant handicapé. Entre autres, l'émergence d'une plus grande reconnaissance des droits de la personne handicapée a modifié grandement la stratégie de l'État à l'égard de ce groupe de citoyens. Après une période de concentration des individus handicapés dans des institutions, l'État cherche maintenant à se départir de cette fonction pour la transmettre aux structures familiales et communautaires. La famille d'origine ainsi que la famille d'accueil reprennent toute leur importance pour l'État. Elles deviennent une solution peu coûteuse pour accueillir et maintenir dans la communauté tout citoyen ne répondant pas aux normes établies.

Également, Marinelli et Dell'Orto (1984) soulignent que les professionnels de la santé ont encouragé le phénomène de désinstitutionnalisation et de maintien de l'enfant handicapé à son domicile. Ils ont renforcé la tendance des années soixante-dix selon laquelle la personne handicapée se développe mieux dans son milieu familial indifféremment du handicap et des soins qu'elle nécessite. Cela signifie qu'il y a de moins en moins de placement d'enfants en institution et que la mère devient culturellement désignée pour s'occuper de son enfant handicapé. D'ailleurs, tous s'attendent de sa part à un dévouement sans limite pour son enfant. La femme doit endurer non seu- 
lement la douleur d'avoir un enfant ayant des limitations, mais également les pressions de l'équipe multidisciplinaire pour poursuivre à la maison l'intervention thérapeutique, peu importe l'investissement de temps, d'énergie, de stress et d'argent.

Ainsi, les mouvements de désinstitutionnalisation et de maintien à domicile de la personne handicapée encouragent les familles à jouer un rôle qu'on a vainement tenté de faire remplir par des appareils bureaucratiques fort coûteux et souvent peu efficaces. C'est une charge lourde pour la famille nucléaire qui représente un système social relativement petit, isolé et interdépendant. L'impact de cette responsabilité sera important et davantage influencé par des facteurs économiques et sociaux que par le type du handicap de l'enfant (Browne, Kirlin et Watt, 1981; Power et Dell'Orto, 1980).

\section{Influences de la législation}

Pour bien comprendre l'influence des politiques sociales sur la prise en charge de l'enfant handicapé et les choix à faire par la mère, il faut revoir brièvement l'évolution de la législation concernant la personne handicapée. Les années soixante marquent vraiment le début de la reconnaissance des droits de la personne handicapée au Québec. L'élément le plus déterminant est assurément la mise sur pied en 1967 de la commission d'enquête sur la santé et le bien-être social. Le rapport qui découle de la Commission Castonguay-Nepveu décrit les conditions pénibles que doivent subir les personnes handicapées. Celles-ci sont considérées comme des citoyens de seconde zone, ayant un niveau de vie très bas et étant incapables d'intervenir dans la société de façon constructive. Les conclusions de ce rapport soulignent la nécessité d'intervenir afin de contrôler les causes d'apparition des déficiences, de réduire au minimum les conséquences sociales, de favoriser l'intégration sociale des personnes handicapées et de faire une gestion rationnelle des ressources et des services (Fougeyrollas, 1985). Toutes ces réflexions contribuent à la multiplication des organismes de promotion des droits des personnes handicapées et à l'adoption en 1978 de la loi assurant l'exercice des droits des personnes handicapées. Cette loi entraîne la création de l'Office des personnes handicapées du Québec (OPHQ, 1987).

Rapidement, I'OPHQ doit faire face aux demandes sans cesse croissantes de sa clientèle qui vit avec sa famille, souvent dans des habitations non adaptées au handicap de l'enfant. Cette situation rend la tâche quotidienne de la mère plus ardue et contribue à l'augmentation des demandes de services. Comme ces demandes excèdent les capacités de l'Office, cela occasionne la création de listes d'attente importantes. En plus de répondre aux différents besoins de sa clien- 
tèle, l'Office se voit aussi obligé de compenser les carences de certains programmes gouvernementaux existants. Tout cela avec des crédits de développement insuffisants pour répondre aux demandes (Mercure, 1989). Malgré la diversité des programmes d'aide disponibles, ceux-ci comblent à peine les besoins de base des personnes handicapées et ils sont insuffisants pour favoriser leur intégration complète (OPHQ, 1987). Dans une tentative de redresser la situation, le gouvernement publie une politique d'ensemble en $1985:$ : À part... égale ». Il s'agit d'un document visant une planification globale de l'ensemble des interventions de la société québécoise en faveur des personnes handicapées. À la suite de cette publication, le mandat de l'OPHQ se concentre sur la coordination du suivi des recommandations et du plan d'action. D'ailleurs, dans les orientations triennales 1988-1991 de À part... égale, il faut souligner la volonté " écrite " de favoriser le développement et la promotion de mesures pour soutenir les familles où vivent des personnes handicapées. Il est proposé de fournir aux parents des renseignements sur la déficience de leur enfant, ses besoins, les ressources et services, et ce, dès la naissance de l'enfant; de s'assurer que les parents reçoivent de la formation; et de voir à la mise en place de mesures de soutien aux parents dans l'exercice de leur rôle auprès de leur enfant handicapé. Malheureusement, une grande partie des mères ayant un enfant handicapé à la maison bénéficient peu d'une aide directe et quotidienne de la part des instances gouvernementales.

Au contraire, I'OPHQ diminue sa participation dans les services directs aux personnes handicapées. II dirigera plutôt ses actions vers une plus grande intégration sociale des personnes handicapées en accordant la priorité au soutien aux familles, à l'utilisation des services scolaires réguliers et à l'intégration au marché du travail. Dans l'avenir, ses nouveaux rôles viseront une coordination des interventions des différents ministères, une analyse des projets de loi, des règlements ou des politiques émises par ces ministères. L'Office vise une plus grande collaboration entre lui et les autres organismes gouvernementaux (Mercure, 1989).

À l'heure actuelle, le Québec ne possède aucune politique globale qui concerne l'ensemble des besoins de ces familles. L'OPHQ trouve cette situation déconcertante et incite le gouvernement à développer un ensemble de mesures impliquant plusieurs ministères et organismes. Aussi, en octobre 1989, l'OPHQ a émis un projet d'orientation intitulé Un air de famille : Le soutien nécessaire aux familles des personnes handicapées. Ce document souligne que l'intégration sociale des personnes handicapées dépend principalement de la qualité de vie qu'elles peuvent trouver dans leur famille. Pour cela, les mesures et les services nécessaires pour le soutien des familles sont définis (OPHQ, 1989). Cependant, aucun échéancier n'est proposé et aucune 
activité concrète pouvant aider les mères d'enfants handicapés n'a été mise sur pied.

Bien sûr, il existe certaines mesures sociales pour tenter d'alléger le fardeau de la mère. Il y a des mesures financières, comme des allocations familiales supplémentaires pour un enfant handicapé, provenant de la Régie des rentes du Québec (plusieurs personnes ne connaissent pas ce service). Également, il existe un programme provincial d'allocation pour couvrir une partie des coûts liés aux services de répit/gardiennage. Cependant, ce programme est administré différemment d'une région à l'autre. D'autres mesures fiscales existent, comme une subvention au moment de l'intégration à la garderie, le remboursement de certaines taxes au niveau fédéral ou encore l'ajout d'un supplément pour le parent vivant de l'aide sociale. Différentes aides techniques (ex. : fauteuil roulant, orthèse ou prothèse) sont payées par la Régie d'assurance-maladie du Québec. Mais I'accès à ces programmes d'aide est limité par des critères d'admissibilité ne correspondant pas toujours à la réalité de la mère. Des limites budgétaires et le manque de disponibilité des ressources humaines spécialisées diminuent I'utilisation de ces services.

II reste différentes façons pour la mère d'avoir du répit. Par exemple, quelques familles d'accueil acceptent de recevoir en dépannage des enfants handicapés durant une courte période. Un nombre limité de lits dans les centres de réadaptation peut aussi accueillir ces enfants. La communauté offre d'autres formes de soutien par l'entremise des organismes de promotion des droits de la personne handicapée ou des associations qui fournissent aux parents des occasions d'échanger et de recevoir du soutien moral (Foucault, 1984).

En résumé, la plupart des services sont dirigés vers les besoins de l'enfant handicapé plutôt que destinés particulièrement à la mère. Même si certaines améliorations des services sont notées, comme en ce qui a trait aux soins à domicile, ces mesures sont insuffisantes et ne répondent qu'à une minorité de personnes. De plus, il faut tenir compte que parallèlement à la mise en œuvre de ces programmes d'aide, des restrictions budgétaires sont imposées. Cette situation amène des coupures de service qui entraînent à leur tour un surcroît de travail à la mère. De plus, même si la communauté tente d'aider la famille de l'enfant handicapé, il faut arriver à l'évidence que les ressources communautaires varient d'une région à l'autre du Québec. Elles sont fragiles, car elles se construisent à l'aide de bénévoles et de subventions parfois éphémères. Ces réseaux d'entraide sont très importants, mais aucun d'entre eux ne fournit des services de répit pour la mère de façon régulière et à long terme. Ils aident les parents, mais ne compensent pas les coûts supplémentaires qu'entraîne le handicap de l'enfant. 


\section{Relation mère-professionnels}

La collaboration des professionnels auprès de la mère peut l'aider à garder le moral, à apprendre à faire face aux tensions quotidiennes, à trouver de nouvelles ressources d'assistance et à atteindre une harmonie maritale (Mallory, 1986). Cependant, Milisap (1988) tout comme Shapiro (1981) soutiennent que les besoins de la famille sont souvent ignorés par les professionnels, incluant l'intervenante ou l'intervenant social! En effet, la famille est régulièrement perçue comme source potentielle de service plutôt que comme ayant besoin d'aide. Les nombreuses demandes adressées à la famille affectent chacun des membres et contribuent à augmenter le risque de voir se développer d'autres problèmes. Ces difficultés peuvent aussi nuire au processus de recouverment ou de réadaptation de l'enfant handicapé (Shapiro, 1981).

Également, la mère doit se soumettre et dépendre des décisions de l'équipe médicale. À propos, les écrits sur le sujet soulignent que les relations des professionnels de la santé avec les parents ne sont pas toujours faciles. Thomas (1982) mentionne l'existence d'une relation de pouvoir inégale entre les deux parties. Pour cet auteur, les professionnels ont tendance à se percevoir comme des experts, $c^{\prime}$ est-à-dire ayant plus de compétence et d'expérience que les parents. Ils considèrent souvent la famille comme nuisant au processus de réadaptation et il leur arrive de ne pas la consulter au moment de prises de décision (Vash, 1981). Malheureusement, la plupart des parents soulignent que les professionnels ne sont pas disponibles et ne répondent pas à leurs attentes. À ce propos, Mallory (1986) signale que les interactions entre la famille et le réseau formel ne sont pas toujours couronnées de succès. Pour Kazak (1986), ce sentiment d'insatisfaction pourrait provenir du fait que l'intervention du professionnel se concentre sur l'enfant, en négligeant les besoins de la famille.

Millsap (1988) soupçonne aussi que la relation entre les professionnels et les parents représente un facteur lié à la surcharge. À ce propos, elle soutient que les professionnels œuvrant auprès de l'enfant handicapé peuvent, par leurs comportements et par leurs demandes, influencer la perception qu'ont les parents de la surcharge. Elle suggère que les contacts parents-professionnels deviennent souvent des événements stressants, car ceux-ci reçoivent clairement la confirmation que leur enfant est handicapé. Pour eux, les séances de thérapie de l'enfant renforcent le constat de ses limites fonctionnelles. Un conflit peut alors facilement naître, surtout si la mère, de son côté, se sent isolé de l'équipe et se perçoit comme ignorante et mal informée. Elle éprouve de la difficulté à rassembler toutes les visions fragmentées de son enfant provenant de chaque professionnel et à se faire une idée 
sur le fonctionnement de celui-ci. Selon Lipsky (1987), le sentiment qu'éprouvent les parents d'être incompétents à l'égard de la prise en charge de leur enfant handicapé est une réaction naturelle au handicap, mais si un tel sentiment se prolonge, il peut être une conséquence directe d'un manque d'aide professionnelle appropriée.

\section{Perception des travailleuses et travailleurs sociaux}

Afin de mieux connaître la perception qu'ont les travailleuses et travailleurs sociaux de la situation de la femme ayant un enfant handicapé, une enquête a été faite auprès de six praticiens ( 4 femmes et 2 hommes) travaillant dans le milieu hospitalier pédiatrique (Tétreault, 1989). Cette étude fait ressortir que la mère est perçue comme la porte d'entrée de la famille. Elle est le membre de la famille qui a la tâche la plus lourde à porter. Elle représente le pilier et le pivot sur lequel tout le monde s'appuie. Toujours selon ces praticiens, la mère possède le plus d'emprise sur la situation et semble s'y adapter plus rapidement que tout le reste de la famille. Naturellement, la femme est plus présente que le père et quitte son emploi plus facilement que celui-ci afin de se consacrer entièrement à son enfant. Une disparité des rôles est présente dans le couple. La mère aura tendance à assumer seule toute cette charge, car elle ne veut pas imposer ces responsabilités à son conjoint. Celui-ci aura tendance à adopter une attitude traditionnelle et à s'éloigner de l'enfant.

Selon les personnes interrogées, la mère possède des capacités d'organisatrice très importantes. Elle encourage, soutient, rassure la famille tout en faisant des démarches pour obtenir des soins appropriés à son enfant. Malheureusement, la mère vieillit plus vite et présente souvent des signes d'épuisement chronique. Ses comportements à l'égard de l'enfant handicapé l'amènent à s'isoler. Elle attend d'être en panne avant de chercher de l'aide et du soutien. D'ailleurs, les répondants et répondantes signalent la difficulté de la mère à demander de l'aide et à confier les soins de son enfant à quelqu'un d'autre.

De façon générale, les travailleuses et travailleurs sociaux analysent la situation d'un point de vue familial plutôt qu'individuel. Ils peuvent fournir de la documentation sur la maladie et indiquer aux parents des ressources disponibles dans la communauté. Ils soulignent l'importance d'expliquer à la famille les événements qui se déroulent autour d'eux. Ils tentent de dédramatiser la situation et de la rendire conforme à la réalité. Mais aucune approche particulière à la mère de l'enfant handicapé n'est préconisée dans la pratique du service social, même si elle demeure la principale intervenante. 


\section{Réflexion féministe}

\section{Intervention non sexiste et féministe}

En examinant l'impact de la présence d'un enfant handicapé sur la mère, le lecteur a noté que les répercussions se font sentir sur la vie sociale, mais également sur la santé physique et psychologique. Qui peut mieux que ces mères démontrer et dénoncer ce problème ? Encore faut-il qu'elles aient suffisamment confiance en leurs propres capacités pour le faire. Considérant cela, cette dernière partie de l'article présente nos réflexions faites en fonction d'une pensée féministe.

L'approche féministe suggère différentes avenues pour les intervenantes auprès de la mère de l'enfant handicapé qui recherchent des moyens plus efficaces pour la soutenir. L'intervention proposée dans cet article se base sur les écrits recensés et sur le principe qu'il existe au départ une inégalité des tâches à l'intérieur du couple, où la plupart des soins de l'enfant handicapé sont sous la responsabilité de la femme. La proposition d'intervention est influencée par l'expérience professionnelle auprès de mères d'enfants handicapés et par l'interprétation de l'approche féministe de la part des deux auteures. Selon notre perception, il est important, dès le départ, de ne pas intervenir uniquement auprès de la mère, mais de s'impliquer auprès du couple. Cette prise de position peut paraître aller à l'encontre de la perspective féministe qui recommande que la femme soit le centre de l'intervention (Cook, 1988). Les principales raisons qui motivent ce choix visent à maximiser l'engagement du père dans le processus d'adaptationréadaptation de l'enfant handicapé, à le sensibiliser à la situation de sa conjointe et à éviter une prise en charge complète des soins de l'enfant par la femme.

Dans un premier temps, une intervention non sexiste est préconisée auprès du couple (Legault, 1980). À la suite de la naissance de l'enfant, les deux parents vivent une période de choc, de douleur et de grande tristesse. Le rôle de la travailleuse sociale consiste à donner du soutien aux parents. Son intervention non sexiste favorise la participation du père, une répartition égale des tâches et l'engagement des deux parents dans le processus d'adaptation. Il est important d'éviter l'isolement de la femme et d'empêcher l'accumulation des responsabilités de l'enfant par la mère. Tout au cours du processus de soutien, la travailleuse sociale incite les membres du personnel hospitalier à enseigner aux deux parents les soins à donner à l'enfant. L'accent est mis sur la participation essentielle des deux parents afin de les sensibiliser à la lourdeur de la tâche pour un seul parent, au risque de surcharge et à l'isolement possible de la inère. De plus, la travailleuse 
sociale apporte une attention spéciale à l'apparition éventuelle de comportements de surprotection de l'enfant et à la perte de l'identité du couple, tout cela pouvant conduire éventuellement à une séparation. À long terme, l'intervention non sexiste tend vers une sensibilisation des parents à l'égard de leurs perceptions des rôles sociaux, des stéréotypes sexuels et du conditionnement social.

Une intervention féministe pourra prendre place dans une deuxième étape (Corbeil, Paquet-Deehy, Lazure et Legault, 1983). Cette intervention encourage les femmes à exprimer leur insatisfaction à l'égard du rôle féminin. Elle favorise la rencontre et l'échange de vues entre ces femmes. Sur le plan individuel, cela diminuera le sentiment de culpabilité, alors que sur le plan collectif les femmes apprendront à mettre en commun leurs problèmes. De plus, cette intervention aide la femme à reprendre confiance en elle, stimule l'atteinte d'une plus grande autonomie et vise l'acquisition d'une indépendance sur le plan de la réalisation et de l'actualisation de soi. La femme apprend à exprimer ses besoins, ses désirs par rapport à la situation vécue, au système hospitalier et aux décisions prises par les médecins. Grâce à cela, elle sera capable de prendre des décisions de façon autonome, en fonction de ses propres besoins. À long terme, la femme vise une autonomie financière et revendique la reconnaissance de ses droits.

Quels pourraient être les rôles de la travailleuse sociale dans cette intervention féministe ? Selon différentes auteures (Corbeil, PaquetDeehy, Lazure et Legault, 1983; Dumas, 1980; Van Der Berg et Cooper, 1986), il existe plusieurs façons pour la travailleuse sociale de s'engager activement en vue d'un changement de la situation de la femme mère d'un enfant handicapé.

Premièrement, son engagement personnel est important. Son rôle, en tant que travailleuse sociale, doit devenir actif, de plus en plus politisé et viser un engagement social pour la cause des femmes. Une prise de conscience du sexisme dans la société et dans le couple doit être faite, tout comme une sensibilisation aux valeurs traditionnelles. Ses actions sont concrètes et peuvent prendre différentes formes, comme développer des mouvements soutenant les mères dans leurs démarches ou encourager la mise sur pied de ressources appropriées favorisant l'atteinte d'une plus grande autonomie. Un rôle de prévention pourrait débuter par la participation de la travailleuse sociale à l'intérieur des cours prénataux, qui sont souvent une première source d'information et de contact avec des mères ayant des grossesses à risque. De plus, le regroupement d'intervenantes ayant une vision féministe de la situation serait un moyen pour exercer des pressions afin de sensibiliser le gouvernement et les autorités qui prennent les décisions ayant des répercussions directes sur la situation des femmes. 
Des manifestations publiques sont nécessaires, que ce soit par des lettres dans les journaux, par des entrevues à la radio et à la télévision.

Tout cela implique un changement de mentalité des autres intervenants et intervenantes, afin d'arriver à une autonomie psychologique et économique de la mère la conduisant vers la prise de pouvoir de sa situation. Ce résultat sera possible s'il y a une relation égalitaire entre elle et les autres professionnels hommes et femmes. Pour cela, la travailleuse sociale encourage la femme à prendre conscience de son importance et à modifier ses comportements de femme-mère. Une démythification des croyances par rapport aux rôles de la mère doit être amorcée auprès des intervenants et intervenantes. D'autres pistes d'action peuvent être explorées dans le domaine du marché du travail, afin de favoriser de meilleures conditions d'emploi pour la femme. L'accessibilité à l'information pour toutes s'avère essentielle. L'autonomie financière peut résoudre certains problèmes vécus par la mère d'un enfant handicapé. Plus de collaboration est nécessaire entre les praticiens sociaux et les professionnels et professionnelles des milieux de la santé. Une sensibilisation aux conditions particulières de la mère doit commencer le plus tôt possible.

\section{Proposition d'une intervention féministe}

À la suite de cette réflexion, une adaptation des prémisses de Van Der Berg et Cooper (1986) est proposée afin de voir comment pourrait s'articuler concrètement l'intervention féministe.

La première prémisse de ces auteures vise à éliminer les fausses dichotomies et les séparations artificielles. Elle dénonce la tendance du pouvoir patriarcal à diviser, à séparer et à isoler les éléments ou les gens. À la lueur de la situation actuelle, il ressort que la prise en charge de l'ensemble des responsabilités concernant l'enfant handicapé revient à la mère. L'isolement est omniprésent dans sa vie. Elle fait face au cloisonnement des multiples professionnels, où chacun est enfermé dans sa spécialité avec son langage technique. En appliquant cette prémisse, l'action féministe encourage une approche généraliste auprès de la mère. Cela implique l'importance de considérer le vécu de la mère avec l'enfant et son milieu de vie particulier. La travailleuse sociale utilise une approche visant à faire tomber et éliminer les barrières. Pour atteindre ce but, les prochaines recherches doivent être plus « engagées ". Également, il faudrait retrouver à l'intérieur même du programme de formation une plus grande réceptivité des étudiantes en service social à cette problématique.

Le deuxième principe envisage la reconceptualisation du pouvoir. II s'agit d'un concept central permettant à la femme d'articuler le 
pouvoir comme une prise en charge de l'action plutôt que comme une domination du plus fort sur le plus faible. Actuellement, la mère vit une dépendance vis-à-vis de l'hôpital, du médecin, du spécialiste, de la famille et des besoins de l'enfant handicapé. Cet état contribue à accroître son isolement, à diminuer son estime de soi et son contrôle sur sa vie. II permet à long terme la viabilité d'une situation impliquant le dominant (domaine médical) qui a la maîtrise de la situation et la dominée (mère) qui subit les décisions. C'est pourquoi la prise en charge de l'action par la mère doit débuter le plus rapidement possible. Cette nouvelle vision du pouvoir redonne à la femme des possibilités d'influencer les événements, d'être capable de contrôler ses émotions et d'utiliser ses habiletés et ses connaissances afin d'exercer un rôle social déterminant. L'augmentation de l'engagement du père, associée à l'établissement de relations égalitaires entre les membres de l'équipe et la mère, devient essentielle. Une répartition plus équitable des tâches entre les membres de la famille est nécessaire. L'autonomie financière et individuelle de la mère (retour aux études, travail...) lui permet également de reprendre la pouvoir. Une autre façon d'appliquer cette prémisse consiste à encourager les chercheuses dans ce secteur à diffuser leurs résultats et à les discuter avec les femmes afin de ne pas limiter leur rôle à celui de répondantes.

La troisième prémisse concerne la valorisation des processus autant que du produit. Cela signifie que la façon dont un objectif est mené à terme est aussi importante que l'atteinte du résultat escompté. Les interventions des professionnels se concentrent sur la survie de l'enfant et sur le processus de réadaptation, tout en négligeant les effets de la situation sur la mère. Les performances de l'enfant en thérapie sont valorisées et l'énergie se concentre sur l'atteinte d'un développement normal. L'application de ce principe féministe vise l'indépendance de la mère et la prise de pouvoir sur le processus de réadaptation. Par exemple, l'action s'oriente vers la participation des praticiens et des praticiennes à donner de l'information de façon périodique à la mère et à décourager sa dépendance à l'égard d'un thérapeute. L'implantation des nouveaux programmes de service doit tenir compte des réalités de la mère. Les travailleuses sociales peuvent encourager la création d'une association de mères d'enfants handicapés et y participer.

Renommer, telle est la quatrième prémisse, qui implique une valorisation de l'expérience personnelle en tenant compte du cadre de référence des femmes. À l'intérieur de la situation actuelle, la mère se sent coupable d'avoir un enfant handicapé. Elle est embarrassée et elle a peur de discuter de ses problèmes avec les autres femmes. La femme éprouve un malaise à parler de la présence du handicap, ce qui favorise son retrait social. Le handicap demeure encore un sujet tabou dans 
notre société. Parmi les actions féministes à entreprendre, il est important de valoriser l'expérience personnelle et de soutenir le choix individuel de la mère. La travailleuse sociale analyse avec les femmes l'influence de la culture sur leur propre vie et sur leur perception des choses. Chacune doit apprendre à se renommer pour bien définir ses fonctions et ses rôles. Une action concrète serait d'organiser des groupes de discussion où la femme partage son vécu, prend conscience d'elle-même et de ce qu'elle peut réclamer.

Le dernier principe féministe de Van Der Berg et Cooper (1986) s'intitule "le privé est politique ". Il désigne une analyse sociale et politique des problèmes rencontrés dans la vie privée. Ces difficultés personnelles sont liées directement à la structure sociale et aux contradictions qui en découlent. D'ailleurs, qui n'a pas entendu dire que l'on doit laver son linge sale en famille ? Le privé est privé. Généralement, il n'est pas recommandé d'étaler au grand jour son privé et encore moins de l'utiliser à des fins publiques ou politiques. Ainsi, chaque femme conserve son expérience de mère d'enfant handicapé, ses sentiments, ses frustrations pour elle et pour son entourage immédiat. L'application de ce principe encourage la travailleuse sociale à entreprendre une éducation politique auprès des mères, afin de montrer que leurs problèmes personnels sont liés à des problèmes de gestion dans le système et des contradictions sociales. Il faut favoriser une reconnaissance des causes socio-économiques des problèmes que vivent ces femmes-mères afin de diminuer la tendance à se blâmer soi-même, à avoir honte et à s'isoler dans son malheur. Pour cela, la travailleuse sociale sert de modèle comme agent de changement et sort du moule traditionnel de conseillère. Sa participation personnelle au sein de sa communauté amènera graduellement des changements dans les mentalités. Le privé est politique, voilà un principe qui se doit d'être appliqué le plus rapidement possible et surtout avec plus de force, sans gêne et sans honte de notre vécu.

\section{Conclusion}

Il est essentiel que la travailleuse sociale modifie son approche pour travailler de pair avec la femme plutôt que de chercher à l'aider à se conformer au modèle de "normalité " que lui impose la société.

L'application graduelle de l'intervention féministe peut contribuer à maintenir la cellule familiale; à briser l'isolement de la mère; à réduire la lourdeur des tâches; à favoriser une plus grande interaction entre le père et l'enfant; et à amener plus de communication dans le couple. Tous ces changements peuvent avoir un effet positif sur le 
mieux-être de la femme qui peut s'épanouir tant physiquement que psychologiquement.

L'intervention féministe proposée dans cet article vise à redonner à la femme un pouvoir qu'elle a enseveli sous la culpabilité et la honte véhiculée par le seul fait d'avoir eu un enfant non conforme aux normes de la société. Ce pouvoir retrouvé permettra à la femme d'établir une relation égalitaire avec son conjoint et de trouver la force pour faire respecter ses droits et ses choix.

Mais il ne faut pas s'illusionner ! Peu d'écrits féministes abordent et analysent la problématique de la femme ayant un enfant handicapé. Est-ce qu'une ignorance de la situation de la femme-mère pourrait expliquer ce manque d'intérêt ou s'agit-il tout simplement d'une illustration que le handicap est toujours un thème tabou et que la femme parle peu de son vécu avec l'enfant handicapé ?

Plusieurs hypothèses peuvent être avancées afin d'expliquer les hésitations des travailleuses sociales à appliquer une intervention féministe. En outre, la peur de l'éclatement du couple peut encourager les travailleuses sociales à se réfugier dans I'approche familiale, plus près des valeurs traditionnelles. Des contraintes du milieu hospitalier, qui est un milieu patriarcal, exercent des pressions sur le personnel en ce qui a trait à la rentabilité et à l'efficacité. Associé à la lourdeur de la tâche des intervenantes, cela contribue à laisser peu de place à l'improvisation et à l'exercice de nouveaux modèles d'intervention. Il n'est pas surprenant que les travailleuses de ces milieux ne soient guère encouragées à favoriser l'autonomie de la mère.

La femme-mère, elle aussi, fait face à beaucoup de pressions vis-àvis de ses rôles. Tout est axé sur le bien-être de l'enfant. Ce fonctionnement qui tend à la normalisation de l'enfant handicapé isole la femme dans un carcan et nuit à son épanouissement. A-t-elle l'énergie pour briser ces barrières ? Pour aller où et pour faire quoi ? Car malgré tout l'enfant handicapé est présent à ses côtés.

Voilà autant $d^{\prime}$ 'interrogations sans réponse, car beaucoup reste à faire dans ce domaine si peu exploré et tellement rempli d'émotivité. $\mathrm{D}^{\prime}$ autres réflexions doivent être menées. La tâche est ardue à cause de la difficile jonction entre une perspective féministe et cette problématique comportant une multitude de facettes comme le handicap, la distribution des rôles, le sens de la maternité, la double tâche, etc. Cependant, comme femme, comme travailleuse sociale, comme mère ou fille, nous ne pouvons rester indifférentes à la situation actuelle. $\mathrm{C}^{\prime}$ est à nous de nous prendre en charge, de nous relever et $\mathrm{d}^{\prime}$ avancer... 


\section{Note}

Les auteures tiennent à remercier Mme Geneviève Martin, professeure à l'École de service social de l'Université Laval, pour son encouragement et ses judicieux commentaires au moment de la rédaction de ce texte.

\section{Références bibliographiques}

Boudreau, F. (1984). De l'asile à la santé mentale. Les soins psychiatriques : Historiques et institutions. Montréal : Éditions Saint-Martin.

BowlBY, J. (1969). Attachment and Loss. New York : Basic Book.

BRESLAU, N. (1983). "Care of disabled children and women's time use ", Medical Care, $21: 620-629$.

Breslau, N., K.S. Staruch et E.A. Mortimer (1982). "Psychological distress in mothers of disabled children ", American Journal of Disabled Child, 136 : 682-686.

BroWNE, J.A., B.A. KIRLIN et S.WATT (eds.) (1981). Rehabilitation services and the social work role : challenge for change. Baltimore : Williams and Wilkins.

BURDEN, R.L. (1980). "Measuring the effects of stress on the mothers of handicapped infants : Must depression always follow ? ", Child Care, Health and Development, 6 : 111-125.

BURLEY, M.M. (1981). " A parent's perspective : you are right, I have no credentials, but... ", dans J.A. Browne, B.A. Kirlin, S. Watt (eds.). Rehabilitation services and the social work role : challenge for change (p. 129-133). Baltimore : Williams and Wilkins.

BUTANI, P. (1970). "Reactions of mothers to the birth of an anomalous infant : a review of the literature ", Maternal Child Nursing Journal, $3: 59-76$.

CHETwYND, J. " Factors contributing to stress on mothers caring for an intellectual handicapped child », British Journal of Social Workers, 15 : 295-304.

CoOK, J.A. (1988). "Who 'mothers' the chronically mentally ill ? ", Family Relations, $37: 42-49$.

Corbeil, C. , A. Paquet-Deehy et G. Lazure (1983). L'intervention féministe, l'alternative des femmes au sexisme en thérapie. Montréal : Éditions SaintMartin.

DAviES, B.M. (1982). The disabled child and adult. London : Baillière Tindall.

DE BILLY, H. (1984). "Des ressources pour les parents ", Carrefour des affaires sociales, $6: 28-30$.

Desroches-Oligny, A. (1980). Martine. Montréal : Éditions Stanké.

DONER, S. (1975). "The relationship of physical handicap to stress in families with an adolescent with spina bifida ", Developmental Medicine and Child Neurology, $17:$ 765-776.

DumAs, B. (1980). Les idéologies et les thérapies féministes, un renversement de perspective. Québec : Le service de recherche C.S.S.Q.

Foucault, P. (1984). Aider... malgré tout. Essai sur l'historique des centres de réadaptation au Québec. Montréal : Les Éditions de l'Association des centres d'accueil du Québec. 
Fougeyrollas, P. (1985). Prévenir, réduire et compenser les conséquences des maladies et traumatismes : déficiences, incapacités et situations de handicaps. Québec : Rapport de la Commission d'enquête sur les services de santé et les services sociaux.

GATH, A. (1977). "The impact of an abnormal child upon the parents ", British Journal of Psychiatry, 130 : 405-410.

GoRDEUK, A. (1976). "Motherhood and the less than perfect child : a literary review ", Maternal Child Nursing Journal, $5:$ 57-68.

HANDLEMAN, J.S. et S.L. HARRIS (1986). Educating the developmentally disabled. Meeting the needs of children and families. San Diego : College Hill Press.

HOLROYD, J. (1974). "The questionnaire on resources and stress : an instrument to measure family response to a handicapped family member ", Journal of Community Psychology, 2 : 92-94.

JeAN, A. (1987). "Baby shop ou le bout de chou éprouvette ", dans F. Saillant, M. O'Neill (éd.) : Accoucher autrement (p. 261-274). Montréal : Éditions Saint-Martin.

KASAK, A.E. et R.S. MARVIN (1984). "Differences, difficulties and adaptation : stress and social networks in families with a handicapped child ", Family Relations, $33: 67-77$.

Kemp, V.H. et D.D. HatmakeR (1989). "Stress and social support in high-risk pregnancy ", Research, in Nursing and Health, $12: 331-336$.

Klaus, M.H. et R.S. KeNNell (1972). "Maternal attachment : Importance of the first postpartum days ", New England Journal of Medicine, 286 : 460-SA.

LamarChe, C. (1987). L'enfant inattendu. Montréal : Boréal.

LAPOINTE-RoY, H. (1987). Charité bien ordonnée. Le premier réseau de lutte contre la pauvreté à Montréal au XIX $x^{e}$ siècle. Montréal : Boréal.

LEGAULT, G. (1980). " D'une approche féministe auprès des femmes en service social », Intervention, $57: 3-9$.

LEVINE, H. et A. ESTABLE (1981). Maternité et rapports de force : essai de critique féministe, théorique et pratique. Ottawa : Université Carleton.

LIPSKY, D.K. (ed.) (1987). Family supports for families with a disabled member. New York : International Exchange of Information in Rehabilitation.

LONSDALE, G. (1978). "Family life with a handicapped child : the parents speak ", Child Care, Health and Development, 4 : 99-120.

MCCUBBIN, M.A. (1989). "Family stress and family strengths : A comparison of single and two-parent families with handicapped children », Research in Nursing and Health, $12:$ 101-110.

MARDINOS, M. (1985). "Modification du rôle de la femme ayant un enfant handicapé ", Santé mentale au Canada, décembre : 29-31.

MARINelLI, R.P. et A.E. Deli'Orto (eds.) (1984). The psychological and social impact of physical disability (2nd edition). New York : Springer Publishing.

Martin-Beausoleil, G. (1982). "L'intervention féministe et le service social individualisé », Service social, $31: 307-329$.

MATTSSON, A. (1980). "Long-term physical illness in childhood : A challenge to psychosocial adaptation », dans P.W. Power, A.E. Dell'Orto (eds.), Role of the family in the rehabilitation of the physically disabled (p. 180-194). Baltimore : University Park Press.

MerCURE, P. (1989). « L'O.P.H.Q. d'hier à demain », Forum, droits et liberté, 12 : 4-5. 
Morı, A.A. (1983). Families of children with special needs. Maryland : Aspen Publications.

Norman, E. et A. MANCuso (1980). Women's issues and social practice. Illinois : F.E. Peacock Publishers.

Office des personnes handicapées du Québec (1982). Historique de l'évolution des services aux personnes handicapées et la situation actuelle au Québec. Dossier $n^{\circ}$ 9, Drummondville, Gouvernement du Québec.

Office des personnes handicapées du Québec (1987). De la Nouvelle-France à l'an 2000. Dossier d'information, volume 9, Québec, Gouvernement du Québec.

Office des personnes handicapées du Québec (1989). Un air de famille... Document d'orientation de l'Office des personnes handicapées du Québec. Drummondville, Gouvernement du Québec.

Power, P.W. et A.E. DelL'ORTo (eds.) (1980). Role of the family in the rehabilitation of the physically disabled. Baltimore : University Park Press.

QUÉNIART, A. (1989a). "Le traitement médical de la maternité : morcellement du corps, effacement des femmes ", dans C. Gendron et M. Beauregard (éd.), L'avenir-santé au féminin (p. 67-81). Montréal : Gaëtan Morin Editeur.

QUÉNIART, A. (1989b). "L'importance du regard de l'Autre dans le rapport des femmes à la maternité ", dans C. Gendron et M. Beauregard (éd.), L'avenirsanté au féminin, (p. 83-102). Montréal : Gaëtan Morin Éditeur.

QUINE, L. et J. PAHL (1985). "Examining the causes of stress in families with severely mentally handicapped children ", British Journal of Social Work, $15: 501-517$.

Ross, M. (1983). Le prix à payer pour être mère. Montréal : Éditions du RemueMénage.

Saillant, F., M. O’Neill (éd.) (1987). Accoucher autrement. Montréal : Éditions Saint-Martin.

SChilling, R.F., M.A. Kirkham, W.H. SnOw et S.P. SChinke (1986). "Single mothers with handicapped children : different from their married counterparts? ", Family Relations, $35: 69-77$.

SHAPIRO, E. (1981). "Family and long-term treatment or care at home : Implications for social work", dans J.A. Browne, B.A. Kirlin, S. Watt (eds.), Rehabilitation services and the social work role : Challenge for change (p. 208221). Baltimore, Williams and Wilkins.

SHAPIRO, J. (1989). "Stress, depression, and support group participation in mothers of developmentally delayed children », Family Relations, 38 : 169173.

SIMONS, R. (1987). After the tears : Parents talk about raising a child with disability, San Diego : Harcourt Brace Jovanovich.

SMITH, R.W. (1986). "Physically disabled children and parental time use », Journal of Leisure Research, 18 : 284-299.

Statistique Canada (1988). "Addenda au quotidien sur l'enquête sur la santé et les limitations d'activités ", Le Quotidien, $3: 20-24$.

TÉtreault, S. (1989). Le travailleur social en milieu de santé. Analyse théorique et expérimentale (travail non publié). Université Laval, École de service social.

THOMAS, D. (1982). The experience of handicap. London : Methuen.

Thorne, B. et M. Yalom (eds.) (1982). Rethinking the family. Some feminist questions. New York : Longman. 
VAN DER BERG, N. et L.B. COOPER (1986). Introduction in feminist vision for social work. Maryland : National Association for Social Workers.

VASH, C.L. (1981). The psychology of disability. New York : Springer Publishing.

ZuCMAN, E. (1982). Famille et handicap dans le monde. Paris : Centre technique national d'étude et de recherches sur les handicaps et inadaptations. 